\title{
Case Report: Chronic Cavitatory Pulmonary Aspergillosis after COVID-19
}

\author{
Sheetal Chaurasia, ${ }^{1 \star}$ Manjunath Thimmappa, ${ }^{2}$ and Saurav Chowdhury ${ }^{1}$ \\ ${ }^{1}$ Department of Pulmonary Medicine, Manipal Hospital Whitefield, Bangalore, India; ${ }^{2}$ Department of Critical Care Medicine, Manipal Hospital \\ Whitefield, Bangalore, India
}

\begin{abstract}
Chronic pulmonary aspergillosis can present in four distinct clinical syndromes, one of which is chronic cavitary pulmonary aspergillosis (CCPA). CCPA is generally associated with a mildly immunosuppressed state or, in immunocompetent patients, with structural lung damage. Severe acute respiratory syndrome coronavirus 2 (SARS-CoV-2) infection has been associated with reactivation of previous quiescent infections such as tuberculosis and invasive fungal infections, but CCPA in a patient with COVID-19 is rarely reported. Here we present the case of a 57-year-old man with CCPA associated with COVID-19 infection in whom latent aspergilloma was most likely activated after SARS-CoV-2 infection. The patient presented with severe COVID and, after initial response to treatment, started to deteriorate due to reactivation of latent aspergilloma to a more aggressive CCPA form. After confirmation of the diagnosis, the patient was initiated on treatment with voriconazole. He showed a good response to treatment with clinicoradiological response. This case also depicts one of the common causes of clinical deterioration in otherwise recovering COVID-19 patients.
\end{abstract}

\section{INTRODUCTION}

Chronic pulmonary aspergillosis (CPA) complicates other respiratory disorders, with the most common form being chronic cavitary pulmonary aspergillosis (CCPA). ${ }^{1}$ Bronchopulmonary aspergillosis tends to be contracted by the inhalation of mycotic spores of Aspergillus species. These spores are ubiquitous in the environment and do not usually cause illness in immunocompetent people or people with normal lungs. ${ }^{2}$ When there is an underlying structural lung disease and/or an immunocompromised status, these spores can lead to four distinct pulmonary syndromes. ${ }^{2}$

Chronic pulmonary aspergillosis is well described in patients with underlying parenchymal lung disease. ${ }^{2}$ There have been infrequent reports of aspergillomas and CCPA in patients with acute COVID-19 and also after recovery from COVID-19.,4 Here we present the case of a 57-year-old man with CCPA associated with COVID-19 in whom latent aspergilloma was most likely activated after severe acute respiratory syndrome coronavirus 2 (SARS-CoV-2) infection. The man did not have a history of immune suppression and activation, and increase in the size of the fungal ball was demonstrated during the course of his hospital stay for treatment of COVID-19, necessitating antifungal treatment.

\section{CASE REPORT}

A 57-year-old man presented with cough, fever, and breathing difficulty for 2 days. His past medical history was significant for previously treated pulmonary tuberculosis (TB) 20 years earlier.

Clinical examination showed a heart rate of $100 /$ minute, respiratory rate of $24 /$ minute, oxygen saturation of $84 \%$, and temperature of $100^{\circ} \mathrm{F}$. Chest examination showed bilateral crackles. Other systems were within normal limits.

Investigations showed a leukocytosis of 8,000 (4,00011,000 cells/microliter), C-reactive protein (CRP) of $54(<10 \mathrm{mg} /$ $\mathrm{dL})$, lactate dehydrogenase (LDH) of 440 (140-280 U/L), ferritin of $800(20-250 \mathrm{ng} / \mathrm{mL})$, and a D-dimer of $548(<250 \mathrm{ng} / \mathrm{mL})$.

*Address correspondence to Sheetal Chaurasia, Department of Pulmonary Medicine, Manipal Hospital Whitefield, Hoodi, Bangalore, India 560066. E-mail: sheetal.utkalit@gmail.com
Liver function tests, renal function tests, and serum electrolytes were normal. COVID-19 reverse transcriptase polymerase chain reaction (RT-PCR) was positive. Chest $x$-ray showed bilateral interstitial infiltrates and a possible cavitatory lesion in the right upper zone. A high-resolution computed tomography (HRCT) chest showed bilateral peripheral ground-glass opacities with right upper lobe cavity measuring $2.3 \times 2.3 \mathrm{~cm}$ containing an opacity inside which was likely a fungal ball (Figure 1). A provisional diagnosis of severe COVID-19 pneumonia with a coexisting aspergilloma was made, and he was admitted for treatment.

The patient was admitted to the intensive care unit and treated with intravenous dexamethasone, Remdisivir, Bipap, anticoagulation, and proning. He responded to treatment and was moved to the general ward on $6 \mathrm{~L}$ oxygen by face mask. However, on day 3 in the ward, he became increasingly hypoxic with oxygen requirement increased to $15 \mathrm{~L}$ /minute by non-rebreather mask.

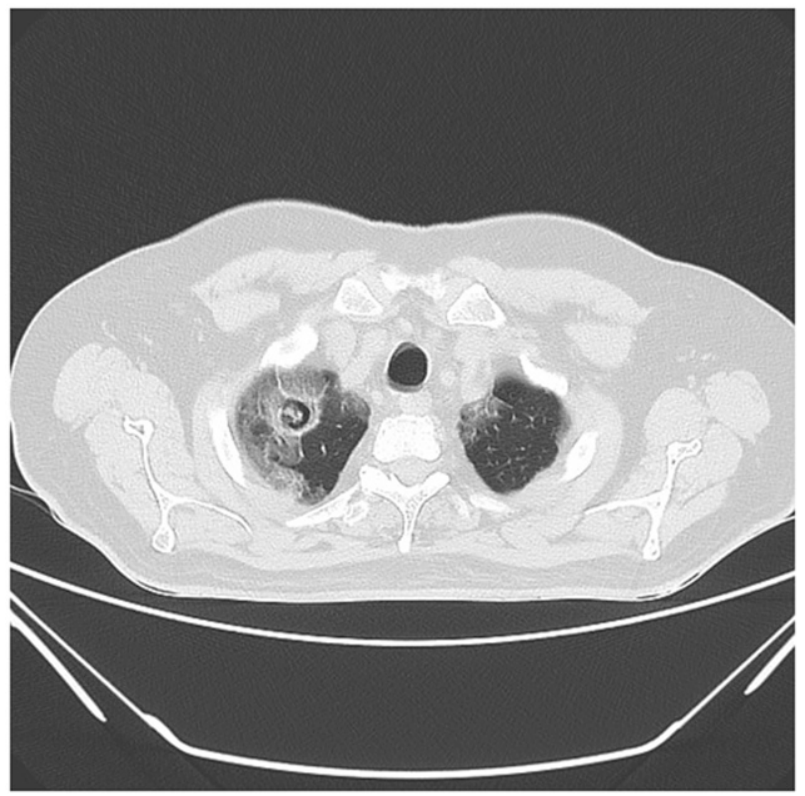

FIGURE 1. High-resolution computed tomography chest showed bilateral peripheral ground-glass opacities with a right upper lobe cavity measuring $2.3 \times 2.3 \mathrm{~cm}$ containing an opacity inside which was likely a fungal ball. 
Repeat laboratory investigation showed leukocytosis of 18,000 (4,000-10,000 cells/ $\mu \mathrm{L})$. However, the CRP, D-dimer, ferritin, and LDH showed a decreasing trend. The blood, sputum, and urine cultures were negative. Sputum was negative for acid-fast bacilli and GeneXpert. A repeat HRCT showed an increase in the right upper lobe cavity size to $3.3 \times 3.2 \mathrm{~cm}$ with an increase in the soft tissue ball to $12 \times$ $8 \mathrm{~mm}$ (Figure 2A). The cavity wall had become more irregular as well. The ground-glass opacities were resolving as compared with the initial HRCT. An increase in the size of the cavity and the opacity with associated irregularity of previously smooth cavity suggested possible invasion by the previously quiescent aspergilloma, likely becoming semiinvasive pulmonary aspergillosis.

An Aspergillus-specific immunoglobulin (Ig)G was positive. Serum galactomannan was $2(<0.5)$. On the basis of the clinical, radiological, and biochemical parameters, he was diagnosed to have chronic cavitating pulmonary aspergillosis. He was started on intravenous voriconazole $300 \mathrm{mg}$ every 12 hours on day 1 followed by 200 mg every 12 hours subsequently, along with the continuation of the dexamethasone, after which the oxygen requirement was reduced and he could be weaned off oxygen and the steroid stopped. Repeat HRCT on day 23 of admission showed disappearance of the previously noted fungal ball, reduction in the size of the cavity, and thinning of the cavity wall (Figure 2B). The voriconazole was planned to continue orally to complete 6 weeks of treatment. He was discharged with a negative COVID-19 RT-PCR on this oral voriconazole. On review 2 months later, he was asymptomatic and was advised to continue the voriconazole for 2 more months.

\section{DISCUSSION}

The diagnosis of CPA requires a combination of the following characteristics: a consistent appearance on thorax imaging, direct evidence of aspergillus infection or immunological response to Aspergillus spp., and exclusion of alternative diagnoses. ${ }^{1}$ Conventionally, CPA should be present for at least 3 months, although that duration may be inferred based on the patient's symptoms or demonstrable progressive radiological abnormality. ${ }^{1}$ In our patient, although there was initially confusion concerning the exact terminology of the disease, the aspergilloma on the initial HRCT with raised Aspergillus-specific immunoglobulin ( $\mathrm{lg}) \mathrm{G}$ as well as the reactivation and increase in size pointed to a chronic rather than an acute condition. His past pulmonary TB most likely resulted in a cavity, which facilitated the formation of an aspergilloma. Because he was immunocompetent, this aspergilloma likely remained quiescent until the infection with SARS-CoV-2. There have been reports of reactivation of TB after SARS-CoV-2 infection, suggesting that the complex immunological processes involved in this infection could lead to reactivation of dormant secondary infections. ${ }^{5}$ In a country such as India with a high burden of active and prior pulmonary TB and its sequalae, the reactivation of silent aspergilloma and progression to a more aggressive form of CPA remains a concern in this pandemic among severe COVID patients receiving high-dose steroids.

SARS-CoV-2-associated pulmonary aspergillosis (CAPA) has been described in COVID-19 patients with acute respiratory distress syndrome. The pathogenesis is incompletely understood but several immunological mechanisms may be responsible for the development of CAPA as well as other fungal infections. ${ }^{6}$ SARS-CoV-2 infection results in the release of danger-associated molecular patterns that act as endogenous signals with a subsequent immune and inflammatory response, resulting in lung injury. Another possibility involves the collateral effects of the host's recognition pathways, which are required for the activation of antiviral immunity. These pathways, while protecting against viruses, may paradoxically contribute to an inflammatory environment that favors fungal pathogenesis. ${ }^{6}$

Despite being described in the literature, the diagnosis of CCPA and CAPA are not straightforward, especially in the setting of the current pandemic. In our patient, even though the cavity and the fungal opacity were found at the time of diagnosis of COVID-19, it was not treated because it was presumed to be an incidental finding without causing the patient any prior discomfort. It was only after the patient's clinical condition deteriorated after initial recovery and a repeat thoracic imaging demonstrating an increase in the size of the cavitatory lesion that the possibility of invasive or chronic-invasive aspergillosis considered.
A

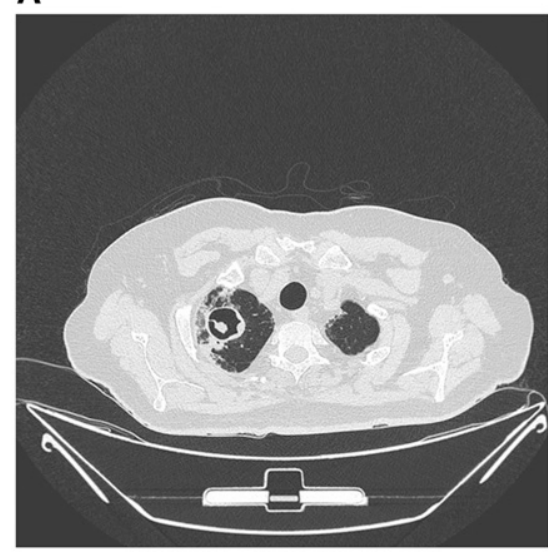

B

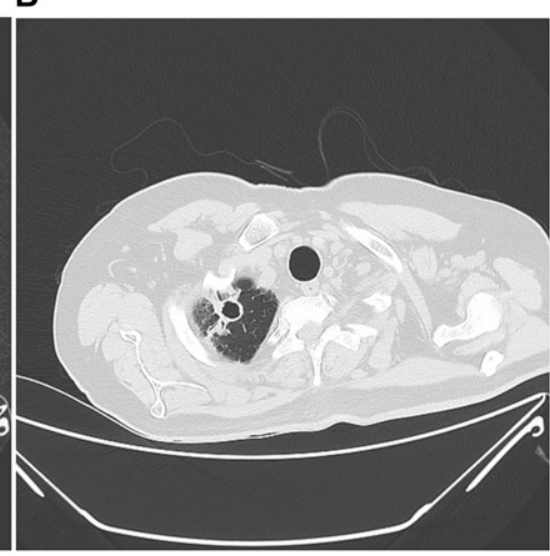

FIGURE 2. (A) High-resolution computed tomography (HRCT) thorax showing an increase in the size of the cavitatory lesion as well as the opacity inside it. (B) HRCT thorax showing the decreasing size of the cavitatory lesion with a resolved inner opacity. 
A minimum of 4 to 6 months of oral triazole therapy is recommended for the treatment of CCPA. ${ }^{1}$ In our patient, we started with intravenous voriconazole because he was acutely unwell at the time of initiation of therapy, followed by a switch to oral after he improved.

CCPA is rarely reported in COVID-19. Pulmonary aspergillosis has been reported in severe COVID-19 in varying degrees, but cavitation associated with Aspergillus infection is reported only in a handful of cases. ${ }^{7}$ This case is also important because the burden of tuberculosis is high in India. With a large number of cases with sequalae in the form of fibrocavity and aspergilloma. Such cases after contracting COVID-19 are at a higher risk of getting a more aggressive form of aspergillus infection

The diagnostic challenges associated with these cases include difficulty obtaining a microbiological diagnosis, heavy reliance on indirect markers such as immunological markers or radiological findings, and possible need for empirical antifungal therapy in the event of negative test results. The presence of elevated Aspergillus-specific IgG, however, can represent either active or prior infection. ${ }^{8}$ Its optimal cutoff level in different geographic areas also remains indeterminate. ${ }^{9}$ It has been found that baseline Aspergillus-specific lgG levels in an Asian population with intermediate TB burden can be expected. ${ }^{10}$ Therapeutic challenges include poor clinical outcomes associated with severe COVID-19, which is worsened by the progressive cavitation and lung damage.

Determinants of clinical outcome include general clinical status of the patient, comorbidities, stage at which the diagnosis of aspergillosis is confirmed, and host response to the treatment

We wish to highlight the possibility of superadded invasive fungal infections in seemingly immunocompetent individuals with COVID-19. SARS-CoV-2 infection and treatment with steroids could play a role in the reactivation of previously quiescent infections. In a patient with COVID-19 who deteriorates after initial improvement, it may be worthwhile to look for an underlying secondary infection, as was found in our case. Treatment of CCPA can lead to favorable outcomes in the setting of early recognition and prompt treatment of the condition.

Received June 21, 2021. Accepted for publication November 3, 2021.

Published online November 24, 2021.
Acknowledgments: The American Society of Tropical Medicine and Hygiene has waived the Open Access fee for this article due to the ongoing COVID-19 pandemic and has assisted with publication expenses.

Authors' addresses: Sheetal Chaurasia and Saurav Chowdhury, Department of Pulmonary Medicine, Manipal Hospital Whitefield, Bangalore, India, E-mails: sheetal.utkalit@gmail.com and saurav.chowdhury@ manipalhospitals.com. Manjunath Thimmappa, Department of Critical Care Medicine, Manipal Hospital Whitefield, Bangalore, India, E-mail: manjut1110@yahoo.co.in.

This is an open-access article distributed under the terms of the Creative Commons Attribution (CC-BY) License, which permits unrestricted use, distribution, and reproduction in any medium, provided the original author and source are credited.

\section{REFERENCES}

1. Denning DW, Cadranel J, Beigelman-Aubry C, Ader F, Chakrabarti A, Blot S, Ullmann AJ, Dimopoulos G, Lange C; European Society for Clinical Microbiology and Infectious Diseases and European Respiratory Society, 2016. Chronic pulmonary aspergillosis: rationale and clinical guidelines for diagnosis and management. Eur Respir J 47: 45-68.

2. Graham KG, Nasir A, 2019. Chronic cavitary pulmonary aspergillosis: a case report and review of the literature. Am J Case Rep 20: 1220-1224.

3. Patti RK, Dalsania NR, Somal N, Sinha A, Mehta S, Ghitan M, Seneviratne C, Kupfer Y, 2020. Subacute aspergillosis "fungal balls" complicating COVID-19. J Investig Med High Impact Case Rep 8: 2324709620966475.

4. Kakamad FH, et al., 2021. Post COVID-19 invasive pulmonary aspergillosis: a case report. Int J Surg Case Rep 82: 105865.

5. Garg N, Lee YI, 2020. Reactivation TB with severe COVID-19. Chest 158: A777.

6. Hoenigl M, 2020. Invasive fungal disease complicating coronavirus disease 2019: when it rains, it spores. Clin Infect Dis 73: e1645-31648.

7. Kumar A, Layek A, Gupta RK, Bala M, Akhlesh, 2021. Aspergilloma in post COVID-19 patient. Arch Pulmonol Respir Care 7: 18-19.

8. Lass-Florl C, Samardzic E, Knoll M, 2021. Serology anno 2021 -fungal infection: from invasive to chronic. Clin Microbiol Infect. Available at: https://doi.org/10.1016/j.cmi.2021.02.005.

9. Richardson MD, Page ID, 2016. Aspgillusserology: have we arrived yet? Med Mycol 55: 48-55.

10. Lee M-R, et al., 2020. Seroprevelence of Aspergillus IgG and disease prevalence of chronic pulmonary aspergillosis in a contry with intermediate burden of tuberculosis: a prospective observational study. Clin Microbiol Infect 26: 1091.e1-1091.e7. 